Fetoplacental Ratio and Fetal Wellbeing

\title{
Comparison of Fetoplacental Ratio in Normal Pregnancy and PIH and Its Association with Fetal Wellbeing
}

Hayam Fathy Ahmed Eittah, Lecturer

Maternal and Newborn Health Nursing, Faculty of Nursing, Menofia University

Tahany El-Sayed Amr, Lecturer

Maternal and Newborn Health Nursing, Faculty of Nursing, Menofia University

Gamila Gaber Ayoub, Lecturer

Maternal and Newborn Health Nursing, Faculty of Nursing, Menofia University

\begin{abstract}
Background: Placenta is required for maintaining pregnancy and promoting normal foetal development. The placenta functions diversely to support the growth of the foetus, interacts with the two individuals, the mother and the developing foetus. Pre-eclampsia and eclampsia leads to various changes in the placenta and its ratio with baby weight with the consequent affection of the fetus. Objective: Comparison of fetoplacenta ratio in normal pregnancy and PIH and it's association with fetal wellbeing. Subjects: The sample was 60 women taken from labor unit; 30 women were normal pregnancy and take code number (1). Other 30 women were pregnancy with PIH and take code number (2). Tools: The tools used in data collection were (Interviewing questionnaire, Anthropometric measurement sheet and Apgar scoring sheet). Results: the mean of placental weight was within normal in both groups $(1,2)$ and there was a slight increase in the mean placenta weight in women with PIH group (2) (608.3 $\pm 53.5 \mathrm{gm})$ compared to $(508.3 \pm 102.6 \mathrm{gm})$ in normal pregnancy group (1). The mean weight of babies in group (1) was $2783.3 \pm 477.1 \mathrm{gm}$. Compared to (3066 \pm 402.5 gm) in group (2). Also there was slight increase in fetoplacental ratio in normal pregnancy group $\left(p<0.05^{*}\right)$. The babies of group one showed higher Apgar scores in 1st and 5th minute of delivery than babies of group two. Conclusion: The fetal outcome was good in normal pregnancy group than pregnancy with PIH group and there was an increase in fetoplacental ratio in normal pregnancy group.
\end{abstract}

\section{Introduction}

Placenta is essential for normal fetal development and failure of the placenta can result in fetal problems because of growing evidence for a correlation of placental weight 